\title{
Effect of timing of hip extension assistance during loaded walking with a soft exosuit
}

\author{
Ye Ding ${ }^{1,2+}$, Fausto A. Panizzolo ${ }^{1,2+}$, Christopher Siviy ${ }^{1,2}$, Philippe Malcolm ${ }^{1,2}$, Ignacio Galiana ${ }^{1,2}$,
} Kenneth G. Holt ${ }^{3}$ and Conor J. Walsh ${ }^{1,2^{*}}$

\begin{abstract}
Background: Recent advances in wearable robotic devices have demonstrated the ability to reduce the metabolic cost of walking by assisting the ankle joint. To achieve greater gains in the future it will be important to determine optimal actuation parameters and explore the effect of assisting other joints. The aim of the present work is to investigate how the timing of hip extension assistance affects the positive mechanical power delivered by an exosuit and its effect on biological joint power and metabolic cost during loaded walking. In this study, we evaluated 4 different hip assistive profiles with different actuation timings: early-start-early-peak (ESEP), early-start-late-peak (ESLP), late-start-early-peak (LSEP), late-start-late-peak (LSLP).
\end{abstract}

Methods: Eight healthy participants walked on a treadmill at a constant speed of $1.5 \mathrm{~m} \cdot \mathrm{s}^{-1}$ while carrying a $23 \mathrm{~kg}$ backpack load. We tested five different conditions: four with the assistive profiles described above and one unpowered condition where no assistance was provided. We evaluated participants' lower limb kinetics, kinematics, metabolic cost and muscle activation.

Results: The variation of timing in the hip extension assistance resulted in a different amount of mechanical power delivered to the wearer across conditions; with the ESLP condition providing a significantly higher amount of positive mechanical power $\left(0.219 \pm 0.006 \mathrm{~W} \cdot \mathrm{kg}^{-1}\right)$ with respect to the other powered conditions. Biological joint power was significantly reduced at the hip (ESEP and ESLP) and at the knee (ESEP, ESLP and LSEP) with respect to the unpowered condition. Further, all assistive profiles significantly reduced the metabolic cost of walking compared to the unpowered condition by $5.7 \pm 1.5 \%, 8.5 \pm 0.9 \%, 6.3 \pm 1.4 \%$ and $7.1 \pm 1.9 \%$ (mean \pm SE for ESEP, ESLP, LSEP, LSLP, respectively).

Conclusions: The highest positive mechanical power delivered by the soft exosuit was reported in the ESLP condition, which showed also a significant reduction in both biological hip and knee joint power. Further, the ESLP condition had the highest average metabolic reduction among the powered conditions. Future work on autonomous hip exoskeletons may incorporate these considerations when designing effective control strategies.

\section{Background}

Exoskeletons have been designed to augment the performance of human locomotion for able-bodied individuals [1-13] or to assist patients affected by different pathologies in rehabilitation and daily activities [14-25]. Positive accomplishments including reductions in energetic

\footnotetext{
* Correspondence: walsh@seas.harvard.edu

${ }^{\dagger}$ Equal contributors

'John A. Paulson School of Engineering and Applied Sciences, Harvard University, 29 Oxford Street, Cambridge, MA 02138, USA

${ }^{2}$ Wyss Institute for Biologically Inspired Engineering at Harvard, 3 Blackfan

Circle, Boston, MA 02115, USA

Full list of author information is available at the end of the article
}

cost of walking, have been achieved in the past 2 years by means of autonomous or tethered systems [5-9, 26-30].

Among these, the majority of the assistive devices that achieved a net reduction in metabolic cost during walking (compared to walking without wearing the device) provided assistance only to the ankle joint [6-9]. Only one device provided assistance to both the ankle and hip joints and achieved metabolic reduction (compared to walking with the device unpowered and removing the equivalent weight of the device from the payload) [30]. This might be due to the fact that the ankle contributes significantly to forward propulsion [31] and can be approximated as a single degree of freedom joint. Further, 
it has been hypothesized that biological power generation at the hip is more costly in terms of metabolic rate [32], since it relies more on the contractile muscle fascicle for power production. In contrast, the ankle joint benefits from the passive elastic properties of the Achilles tendon $[32,33]$. Consequently, it has been proposed that providing external power to the hip joint via a wearable robot could provide a larger reduction in metabolic cost than providing the same amount of power at the ankle joint [32].

A number of different research groups have investigated the effects of powering the hip joint via simulation [34-36] or experimental studies [4, 5, 21, 24, 37]. Giovacchini et al. [4] developed an autonomous robotic hip exoskeleton that can deliver flexion-extension torques to the wearer but no detailed biomechanical or physiological evaluation has been presented to date. A study by Lenzi et al. [21] showed that actuating a wearer's hip with a fraction of the nominal torque profile using a modified treadmill-based lower extremity exoskeleton, can reduce both hip flexor and ankle plantar flexor muscle activation. Lewis and Ferris [37] have reported reduced biological joint torques with a pneumatically powered hip exoskeleton, suggesting that humans alter the net muscle moment at the hip when walking with hip assistance so that the net joint moment is the same regardless of the external actuation under same task constraints. The Stride Management Assist system [5] was designed to increase the walk ratio (step length/cadence) when applying a $3 \mathrm{~N} \cdot \mathrm{m}$ flexion and extension torque at the hip. With this autonomous hip exoskeleton, the authors reported a reduced metabolic cost of $\sim 7 \%$ compared to walking with the exoskeleton unpowered. Ronsse et al. [24] designed a treadmill-mounted hip exoskeleton controlled by means of adaptive oscillators and tested its effect on metabolic cost of walking. They reported a metabolic reduction of $\sim 18 \%$ compared to walking with the exoskeleton unpowered when providing an assistive torque corresponding to $100 \%$ of the average biological torque produced by the hip during walking. Last, previous work from our group [28] compared the effect of hip extension assistance with multi-joint assistance (ankle plantarflexion and hip extension assistance) during loaded walking on metabolic cost with a tethered multi-joint actuation platform. We found an average reduction of $4.6 \%$ compared to walking with the unpowered soft exosuit when delivering an average peak force of $95 \mathrm{~N}$, resulting in an average peak torque of $16 \mathrm{~N} \cdot \mathrm{m}$ to the hip joint [28]. However, none of the previous research has studied the biomechanical and physiological effects of different assistive profiles at the hip joint.

Understanding the effects of different hip assistive profiles is a fundamental step in the process of designing assistive devices and controllers that can provide better performance in terms of metabolic cost. While there is promising early work in assisting the hip joint, there is limited literature including studies exploring the effects of the timing and magnitude of assistance in a systematic way as it has been done in studies investigating the ankle joint $[6,7,38,39]$.

Therefore, the goal of the present study was to investigate how onset and peak timings of hip extension assistive profiles affected the positive mechanical power delivered by the soft exosuit and its effect on biological joint power and metabolic cost. Previous studies involving ankle exoskeletons $[7,39]$ showed the importance of actuation timing as it affects the positive mechanical power delivered to the wearer and the metabolic cost of walking. This is also likely to be a key factor for hip exoskeletons as shown by a recent simulation study [40] exploring the optimal hip retraction timing for assisting the hip joint. Thus, we designed four different assistive profiles with the aim of analyzing the effect of two specific features: i) the onset timing and ii) the peak timing during stance. While the timing was varied and the magnitude of peak force was kept constant, the delivered positive mechanical power varied due to the duration of the assistance and the different hip velocities. Assuming no major kinematic changes imposed by our hip extension assistance (as demonstrated by our previous work [28, 30]), a longer period of assistance (early onset timing) and a better synchronization of actuation with a period of high hip joint velocity can deliver more positive mechanical power to the wearer. Thus, we explored different timings to regulate the amount of positive mechanical power delivered during the swing and stance phase, both of which are related to the metabolic cost of walking [41, 42]. We tested the 4 different assistive profiles on eight healthy participants wearing the soft exosuit. Loaded walking was investigated because it is a common task that puts severe mechanical and physiological challenges on human locomotion, thus representing an interesting gait condition to explore the effect of a lower limb exoskeleton on the users. Also, the ability to carry substantial loads is required in many professions that execute highly physically demanding tasks associated with their gait [43].

\section{Methods}

\section{Soft exosuit and actuation platform}

For this study, we used a hip soft exosuit and a programmable multi-joint actuation platform that we previously reported in $[28,29,44]$. The textile components of the hip exosuit (Fig. 1) consisted of a spandex base layer (mass: $222 \mathrm{~g}$ ), a waist belt (mass: $251 \mathrm{~g}$ ), 2 thigh braces (mass: $2 \times 76 \mathrm{~g}$ ) and 2 inertial measurement unit (IMU) straps (elastic bands that hold IMUs on the anterior part of the thigh; mass: $2 \times 35 \mathrm{~g}$ ). Compared to 


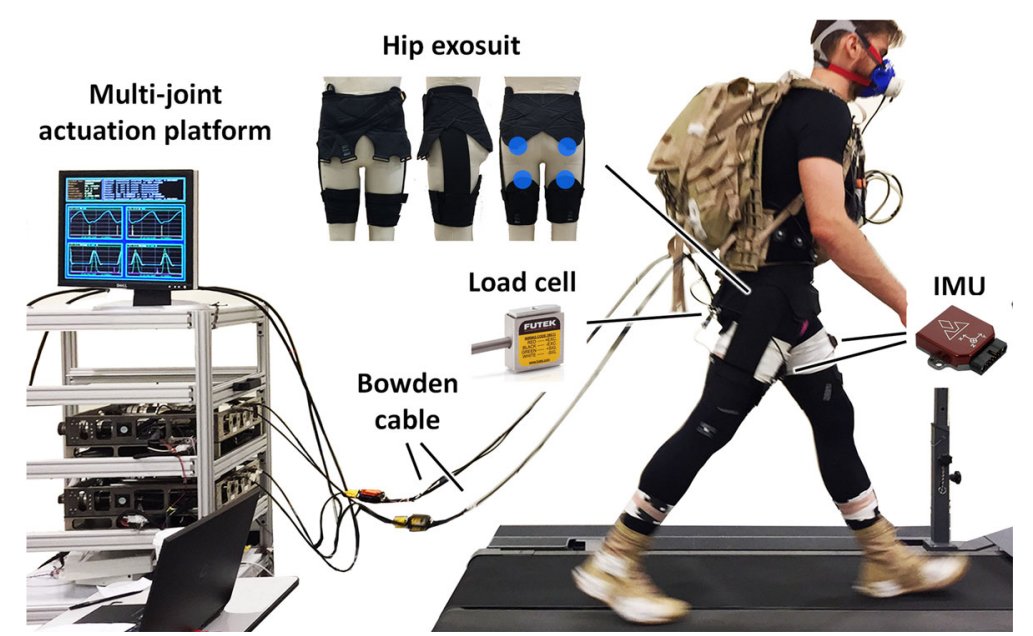

Fig. 1 Experimental setup with a participant wearing a soft exosuit that assists hip extension via Bowden cable. The assistive force is transmitted from the multi-joint actuation platform (on the left) to the wearer

our previously described hip soft exosuit [28], the components used for this study were constructed with a woven fabric with reinforcement webbing and neoprene was added to the waist belt close to where it interfaced to the iliac crest of the wearer. The multi-joint actuation platform was a tethered system designed to provide biologically inspired torques to multiple joints through Bowden cables either individually or simultaneously [44].

Briefly, in this Bowden cables-based force transmission, the sheath covering the inner cable is fixed to the actuator frame and the inner cable is connected to the actuation carriage on the ball screw. The actuator moves the carriage back and forth to either generate tension forces in the soft exosuit (pulling the cable) or to become fully transparent (feeding out cable so it becomes slack). The other end of the Bowden cable sheath is connected to the attachment point at the bottom of the waist belt and the inner cable is connected to the top center of the thigh brace on the back of the leg (Fig. 1). By pulling the cable, the actuation platform shortens the distance between the 2 attachment points and delivers a controlled force in parallel to the wearer's hip extension muscle group thus generating an assistive torque around the hip joint.

\section{Sensing and control}

Our system used an IMU-based iterative controller to deliver a consistent force profile in synchrony with the wearer's individual joint kinematics to accommodate the variability of hip kinetics and kinematics across subjects [29]. One IMU (VectorNav Technologies, Dallas, Texas, USA; mass: $13 \mathrm{~g}$ ) was attached to the front of each thigh to detect the maximum thigh flexion angle to segment the stride. The algorithm identified the first positive thigh angle peak (corresponding to maximum hip flexion angle) after a negative thigh angle peak (corresponding to maximum hip extension angle) as the maximum hip flexion point. Stride time was measured by the controller as the time between 2 consecutive maximum hip flexion events. Load cells (LSB200, Futek Advanced Sensor, USA; mass: $16 \mathrm{~g})$ were placed in series with the Bowden cables to monitor the delivered force. The simulated trapezoidal position profile of the actuator was calculated based on the desired force, average hip joint kinematics and suit stiffness [29]. This position profile was scaled by the average stride time calculated from the previous 2 steps and commanded to the actuator. The iterative controller then automatically adjusted the offset and magnitude of the position profile based on the measured pretension force and peak force from the previous stride. By continuously correcting the actuator position profile, the desired force profile could be achieved without requiring an accurate initial simulated position profile. This iterative control structure allowed us to robustly control the timing and magnitude of the desired force profiles. Detailed controller performance and evaluation are described in [29].

\section{Assistive force profiles}

The goal of the present study was to investigate the effect of onset and peak timings for a given level of hip extension assistance with a soft exosuit. In order to compare the onset timing effects, we used 4 different assistive force profiles: 2 profiles with early onset timing to assist hip joint during terminal swing and 2 profiles with late onset timing initiated during early stance (Fig. 2). Early onset timing (around $90 \%$ of the gait cycle) is coincident with the onset of hip extension just prior to heel strike, and the late onset timing (around $0 \%$ of the gait cycle) is coincident with heel strike. Similarly, two of the profiles had early peak timing and the other 2 had 


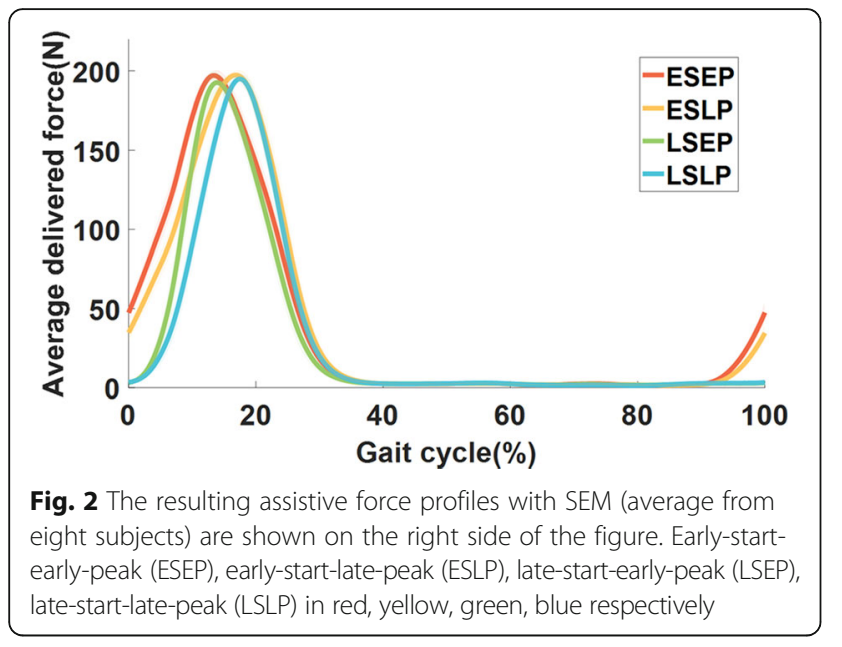

late peak timing. Early peak timing (around $13 \%$ of the gait cycle) is approximately coincident with peak hip power [45] and late peak timing (around $17 \%$ of the gait cycle) was chosen as a first exploration to exploit the higher hip velocity present at that point of the gait cycle $[45,46]$, thus delivering a higher mechanical power while keeping a constant peak force magnitude. Hereinafter, the 4 profiles are referred as early-start-early-peak (ESEP), early-start-late-peak (ESLP), late-start-early-peak (LSEP) and late-start-late-peak (LSLP). The designed and tested onset and peak timing of the profiles are shown in Fig. 2.

\section{Participants}

Eight male healthy participants with no previous experience in walking with the present configuration of the soft exosuit (age $29.8 \pm 5.0$ year, mass $82.6 \pm 5.8 \mathrm{~kg}$, height $1.79 \pm 0.05 \mathrm{~m}$, mean $\pm \mathrm{SD}$ ) were recruited for this study. All participants were free from musculoskeletal injuries and other musculoskeletal diseases and provided written informed consent prior to participating in the study. Participants have provided consent for the publication of their images according to the Journal of NeuroEngineering and Rehabilitation policies. The study was approved by the Harvard Medical School Committee on Human Studies.

\section{Testing protocol}

Participants wore the soft exosuit while walking on an instrumented split-belt treadmill (Bertec, Columbus, $\mathrm{OH}$, USA) at a constant speed of $1.5 \mathrm{~m} \cdot \mathrm{s}^{-1}$ while carrying a $23 \mathrm{~kg}$ weighted backpack; these conditions were chosen because representative of a load carrier population such as soldiers and to allow comparisons with previous exoskeletons studies [8].

The protocol was split into a training session and a testing session with at least 2 days in between to avoid fatigue effects. During the training session, the participants familiarized themselves with the soft exosuit and the experimental setup. They walked for 8 randomized 6-minute bouts, experiencing each of the 4 different assistive profiles twice. Participants rested between the conditions according to their own requests. At the beginning of the testing session, a 5-minute standing trial was performed to collect steady-state standing metabolic cost. After an initial walking warmup of 4 minutes (1 minute for each assistive profile), the participants took a rest of 5 minutes. Subsequently, they underwent five 6-minute data collection bouts: the 4 assistive conditions and 1 unpowered condition with the device turned off. The 5 walking bouts were randomized to minimize any fatigue, order and learning effects. Adequate rest on an average of 5 minutes was given between walking bouts to allow physical recovery; the training and testing sessions are outlined in Fig. 3. After each condition, participants provided information about their perceptions of assistive conditions on a visual analogue scale (Additional file 1: Table S1).

In the unpowered condition, the participants still wore the soft exosuit as this was connected to the actuation platform. This choice was taken to avoid repositioning the markers used for kinematic analysis and the associated changes in the backpack location which could have led to increased variability. Nevertheless, to assess the difference in metabolic cost between wearing the textile components of the soft exosuit and wearing normal clothing we performed additional testing on 6 participants (age $29.0 \pm 4.3$ years., mass $75.6 \pm 6.4 \mathrm{~kg}$, height $1.79 \pm 0.04 \mathrm{~m}$, mean $\pm \mathrm{SD}$ ) on a separate day. After collecting a 4-minute standing trial, participants walked

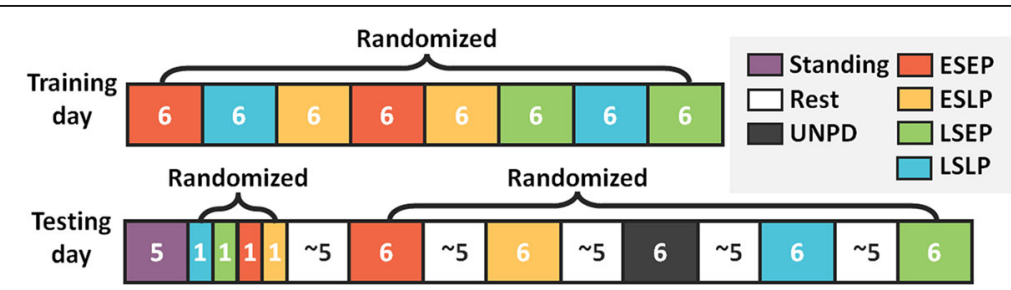

Fig. 3 Testing protocol during training and testing session. Numbers in each block represent the duration of each condition: early-start-early-peak (ESEP), early-start-late-peak (ESLP), late-start-early-peak (LSEP), late-start-late-peak (LSLP) and unpowered (UNPD) in red, yellow, green, blue and black respectively 
under the same testing conditions $\left(1.5 \mathrm{~m} \cdot \mathrm{s}^{-1}\right.$ and $23 \mathrm{~kg}$ of load) for 2 bouts of 6 minutes wearing the soft exosuit or wearing a pair of pants (mass: $715 \mathrm{~g}$ ). These 2 conditions were randomized across the participants.

\section{Data collection and analysis}

Kinematic data were collected through 9-camera Vicon optical motion capture system (Oxford Metrics, Oxford, UK; $120 \mathrm{~Hz}$ ) using 50 markers placed on selected anatomical bony landmarks. Two pairs of additional markers were placed on the right and left proximal and distal attachment points of the Bowden cable. The moment arm of the applied force was calculated continuously across the gait cycle as the normal distance from the line between each pair of markers to the corresponding hip joint center. Ground reaction forces (GRFs) were collected via the instrumented split-belt treadmill. All markers and GRF trajectories were filtered at the same frequency using a zero-lag fourth order low pass Butterworth filter with a $5-15 \mathrm{~Hz}$ optimal cut-off frequency that was selected using a custom residual analysis algorithm which evaluated the difference between the filtered and the unfiltered signals [45] implemented in MATLAB (MATLAB, The MathWorks Inc., USA). Joint angles, joint moments and powers were calculated in the sagittal plane by means of kinematic and inverse dynamics (Visual 3D, C-Motion, Rockville, MD, USA). Joint moments and powers were then normalized by each participant's body mass. An automatic gait event detection algorithm (Visual 3D, C-Motion, Rockville, MD, USA) was used to determine heel strike in order to define gait cycles. To compute the biological components of net joint moment and power during the powered conditions, the actuation platform was synchronized to the Vicon system using a $5 \mathrm{~V}$ signal generated at the beginning of the motion capture data collection. Delivered hip extension moments generated by the soft exosuit during the powered condition were calculated for each participant as the product of the force recorded by the hip load cell and the computed moment arms. Moment arms were defined as the perpendicular distance between the markers on the cable and the respective joint center. The biological joint moments produced during the powered conditions were then calculated by subtracting the moment generated by the soft exosuit at the hip from the net hip joint moment as per [30]. Biological moment was then multiplied by joint velocity to obtain biological power. All data were segmented and normalized to $0-100 \%$ of the gait cycle. Ten strides per condition collected during the last minute of each condition were used for generating mean kinematic and kinetic data for each individual participant, which were subsequently combined to calculate condition mean data.

Metabolic cost was assessed by indirect calorimetry using a portable gas analysis system $\left(\mathrm{K}_{4} \mathrm{~b}^{2}\right.$, Cosmed, Roma, Italy), which enabled the measurement of expired gas concentrations and volumes. Carbon dioxide and oxygen rate were averaged across the last 2 minutes (minutes $4-6$ ) of each walking condition and then used to calculate metabolic rate using the Brockway equation [47]. Net metabolic rate for each condition was obtained by subtracting the standing metabolic power from the walking metabolic power of each condition and then normalizing it by the body mass of each participant. The metabolic reduction was obtained by subtracting the assistive conditions from the unpowered condition. The average metabolic reduction was calculated from the metabolic results of 6 out of 8 subjects. In the 2 subjects not included there were malfunctions in the portable pulmonary gas exchange measurement device during the test which prevented us from using their data.

During the testing session surface electromyographic signals (EMG) from 6 lower limb muscles were measured with a wired system (Delsys, Natick, MA, USA; $2160 \mathrm{~Hz}$ ). The 6 muscles recorded were: rectus femoris (RF), vastus medialis (VM), gluteus maximum (GM), biceps femoris (BF), soleus (SOL), medial gastrocnemius (MG). Electrodes were placed following guidelines in [48]. EMG signals were band-pass filtered (fourth order Butterworth, cut-off $20-450 \mathrm{~Hz}$ ), rectified and low-pass filtered (fourth order Butterworth, cut-off $6 \mathrm{~Hz}$ ) to obtain an EMG linear envelope. EMG signals were normalized by the average of corresponding EMG peaks recorded during the unpowered condition. Linear envelopes for each muscle group were segmented and normalized to each gait cycle. The same 10 strides per condition used for kinematic and kinetic analysis were used for generating average of muscle activation across each stride, which were subsequently combined to calculate condition mean data.

\section{Statistical analysis}

Repeated measures analysis of variance (ANOVA) was conducted across the 4 powered conditions to assess differences in the positive mechanical power delivered by the soft exosuit. Repeated measures ANOVA including 5 conditions (unpowered, ESEP, ESLP, LSEP, LSLP) were used to verify the effect of assistance on positive biological joint powers, peak flexion and extension joint angles (for hip, knee and ankle), as well as peak and average value of the biological hip extension moment and knee extension moment during the first half of the gait cycle. Additional repeated measures ANOVA were also used to verify the effect of assistance on metabolic cost, spatiotemporal parameters and root mean square (RMS) of muscle activation. If a significant main effect was observed $(p<0.050)$, pairwise comparisons were conducted using Tukey's honestly significant difference test similar to [49]. Student's paired $t$-test was performed to assess the differences in net metabolic rate between walking with the textile components of the soft exosuit 
and walking with standard clothing. Linear regression was used to determine correlations between perceptions scores and metabolic cost. Correlation coefficients $(r)$ and significance level $(p<0.050)$, as well as all the statistical analyses were conducted in Matlab (The MathWorks Inc., USA). All the parameters presented in the results section are in the form of mean \pm standard error of the mean (SEM).

\section{Results}

Table 1 summarizes the system performance in controlling the onset and peak timings as well as the peak force of the 4 assistive profiles. The average peak of the assistive force was $197.6 \pm 0.2 \mathrm{~N}$, which results in an average peak of assistive moment of $30.4 \pm 4.7 \mathrm{Nm}$. The average error between designed and measured onset timing and peak timing were within $1 \%$ of the gait cycle. In all powered conditions, the actuation ended at $35.9 \pm 0.6 \%$ of the gait cycle.

The positive mechanical power delivered by the soft exosuit to both limbs in the different conditions was $0.198 \pm 0.003 \mathrm{~W} \cdot \mathrm{kg}^{-1} \quad$ (ESEP), $0.219 \pm 0.006 \mathrm{~W} \cdot \mathrm{kg}^{-1}$ (ESLP), $0.185 \pm 0.009 \mathrm{~W} \cdot \mathrm{kg}^{-1}$ (LSEP), $0.198 \pm 0.006 \mathrm{~W} \cdot \mathrm{kg}^{-1}$ (LSLP), as shown in Fig. 4a. ESLP delivered a significantly higher positive mechanical power with respect to the other powered conditions (ESEP; $p=0.016$, LSLP; $p=0.020$, and LSEP; $p<0.001)$.

In the ESLP condition, the average positive biological knee power was reduced by $0.02 \pm 0.01 \mathrm{~W} \cdot \mathrm{kg}^{-1}(p=0.007)$ and the average positive biological hip power was reduced by $0.06 \pm 0.03 \mathrm{~W} \cdot \mathrm{kg}^{-1}(p=0.007)$ with respect to the unpowered condition. In the ESEP condition, the average positive biological knee power was reduced by $0.04 \pm 0.01 \mathrm{~W} \cdot \mathrm{kg}^{-1}(p<0.001)$ and the average positive biological hip power was reduced by $0.07 \pm 0.02 \mathrm{~W} \cdot \mathrm{kg}^{-1}$ $(p=0.002)$ compared to the unpowered condition. In the LSEP condition, the average positive biological knee power was reduced by $0.03 \pm 0.01 \mathrm{~W} \cdot \mathrm{kg}^{-1} \quad(p=0.002)$. No changes in the average positive biological joint power were observed at the ankle joint $(p=0.584)$. Average positive biological joint power results are presented in Fig. $4 \mathrm{~b}$. Average value of the biological hip extension moment was reduced in all the powered conditions $(p<0.001)$ and peak knee extension moment during the first half of the gait cycle was reduced in the ESEP and in the LSEP conditions $(p=0.009$ and $p=0.044$, respectively), Fig. 5 . No changes in average positive joint moment were observed in the ankle joint $(p=0.432)$.

Standing with the $23 \mathrm{~kg}$ load required $1.52 \pm 0.08 \mathrm{~W} \cdot \mathrm{kg}^{-1}$ and walking in the unpowered condition was $5.92 \pm$ $0.18 \mathrm{~W} \cdot \mathrm{kg}^{-1}$. All powered conditions significantly reduced metabolic cost compared to the unpowered condition $(p<0.015)$ but no significant differences were found among the powered conditions (Fig. 6). Reductions in metabolic rate in the powered conditions were $0.35 \pm$ $0.10 \mathrm{~W} \cdot \mathrm{kg}^{-1}$ (ESEP), $0.50 \pm 0.05 \mathrm{~W} \cdot \mathrm{kg}^{-1}$ (ESLP), $0.37 \pm$ $0.08 \mathrm{~W} \cdot \mathrm{kg}^{-1}$ (LSEP), $0.42 \pm 0.11 \mathrm{~W} \cdot \mathrm{kg}^{-1}$ (LSLP). These values correspond to relative reductions of $5.7 \pm 1.5 \%$, $8.5 \pm 0.9 \%, 6.3 \pm 1.4 \%$ and $7.1 \pm 1.9 \%$ respectively. A small, not statistically significant difference $\left(0.10 \pm 0.14 \mathrm{~W} \cdot \mathrm{kg}^{-1}\right.$, $p=0.509)$ in the net metabolic cost was found between walking with the textile components of the soft exosuit and with a pair of regular pants $\left(5.62 \pm 0.20 \mathrm{~W} \cdot \mathrm{kg}^{-1}\right.$ and $5.52 \pm 0.13 \mathrm{~W} \cdot \mathrm{kg}^{-1}$, respectively).

There were no significant differences in spatiotemporal parameters across the conditions $(p \geq 0.120$, Additional file 1: Table S2). There were no significant changes in peak extension and flexion in all joint angles $(p \geq 0.088)$. Also, no significant changes were found in RMS of muscle activations across the conditions $(p \geq 0.097)$. There was no significant correlation between perception scores and metabolic reduction $(p \geq 0.810, r \leq 0.045)$. Questionnaire results are presented in Additional file 1: Table S1.

\section{Discussion}

The aim of this study was to investigate how the onset and peak timing of hip extension assistance for a given amount of peak force would affect the positive mechanical power delivered by the soft exosuit and its effect on biological joint power and metabolic cost during loaded walking. Results demonstrated that the proposed controller can deliver consistent profiles to the hip joint during loaded walking. The highest amount of positive mechanical power was delivered in the ESLP condition, indicating that a long duration of assistance (early onset timing) together with exploiting higher hip velocity (later peak timing) is a favorable strategy in order to deliver more positive mechanical power to the wearer.

Table 1 Designed and measured peak force, onset timing and peak timing of the four assistive profiles

\begin{tabular}{|c|c|c|c|c|c|c|}
\hline & \multicolumn{3}{|l|}{ Designed } & \multicolumn{3}{|l|}{ Measured } \\
\hline & Peak force $(\mathrm{N})$ & Onset (\%) & Peak (\%) & Peak force $(\mathrm{N})$ & Onset (\%) & Peak (\%) \\
\hline ESEP & 200.0 & 90.0 & 13.0 & $198.1 \pm 0.1$ & $90.2 \pm 0.1$ & $13.3 \pm 0.4$ \\
\hline ESLP & 200.0 & 90.0 & 17.0 & $198.5 \pm 0.1$ & $89.5 \pm 0.2$ & $17.0 \pm 0.4$ \\
\hline LSEP & 200.0 & 0.0 & 13.0 & $197.7 \pm 0.2$ & $0.7 \pm 0.1$ & $13.4 \pm 0.4$ \\
\hline LSLP & 200.0 & 0.0 & 17.0 & $196.0 \pm 0.2$ & $0.2 \pm 0.1$ & $17.9 \pm 0.4$ \\
\hline
\end{tabular}

Early-start-early-peak (ESEP), early-start-late-peak (ESLP), late-start-early-peak (LSEP), late-start-late-peak (LSLP). Data are mean \pm SEM 


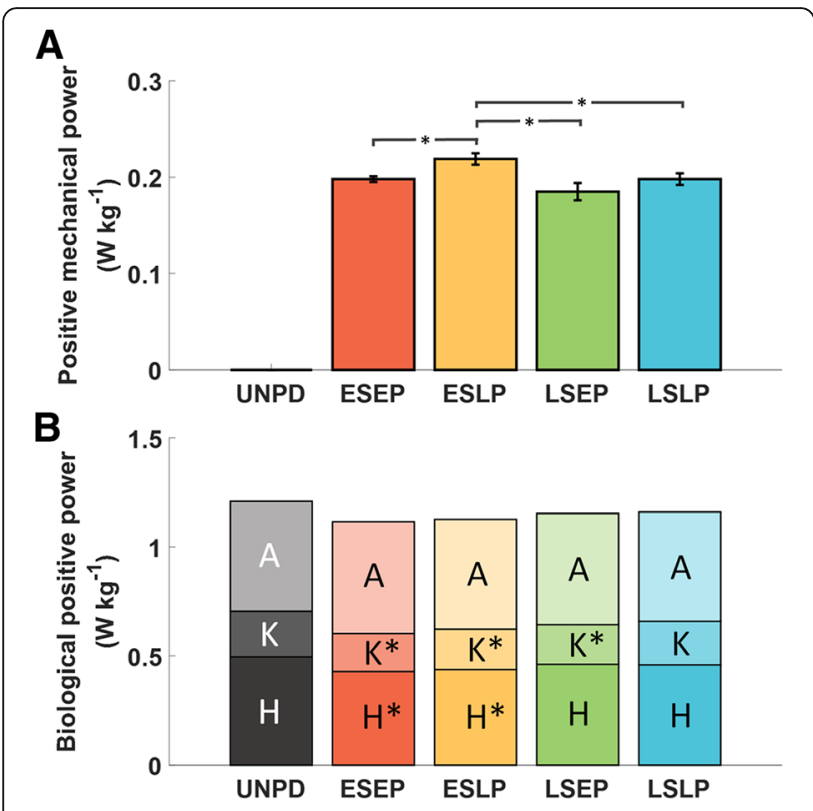

Fig. 4 a Positive mechanical power delivered by the soft exosuit to both limbs. Data are means \pm SEM. The braces with * represent statistically significant differences between two conditions $(p<0.050)$. b Average positive biological joint power of both limbs in the unpowered condition (UNPD) and in the powered conditions. The rectangles with letter $\mathrm{A}, \mathrm{K}, \mathrm{H}$ in each bar represent ankle, knee, and hip positive biological joint power. The small panel on the right corner shows the corresponding assistive profile. Early-start-early-peak (ESEP), early-start-late-peak (ESLP), late-start-early-peak (LSEP), late-start-latepeak (LSLP) in red, yellow, green and blue respectively. The * represents statistically significant differences with the unpowered condition $(p<0.050)$
Biological joint power was reduced at the hip (ESEP and ESLP) and at the knee (ESEP, ESLP and LSEP). It is reasonable to hypothesize that these reductions in biological joint power led, at least in part, to the metabolic reduction, similar to the findings of previous work on an ankle-only and on a multi-joint exoskeleton [9, 28, 30]. Further, the ESLP condition, which achieved the highest metabolic reduction, was one condition that reduced biological joint power for both the knee and the hip joints. This result may indicate that an additional metabolic saving is obtained when the external assistance alters the mechanics of non-assisted joints, thus favorably tuning the musculoskeletal system as a whole $[6,9,28]$ rather than acting on a specific muscle group. The reduction of the biological joint moment during the first half of the gait cycle in the assistive profiles may also have contributed to the metabolic reduction. This is because the biological joint moment is proportional to the muscle forces, and the cost of muscle force production accounts for $\sim 50 \%$ of the metabolic cost of transport in humans [50]. Recent exoskeleton studies have also proposed a reduction in joint moment as an explanation for the decreased metabolic cost $[6,51]$.

Metabolic cost was significantly reduced in all the powered conditions compared to the unpowered condition, confirming the efficacy of the hip assistance in reducing the metabolic cost of loaded walking (Fig. 6). Nevertheless, the lack of statistically significant differences between the powered conditions prevents conclusions on the best assistive profile to optimize the metabolic expenditure.

No significant differences were found in muscle activation, similar to our previous work with a hip actuated soft exosuit [28]. Due to the different factors affecting
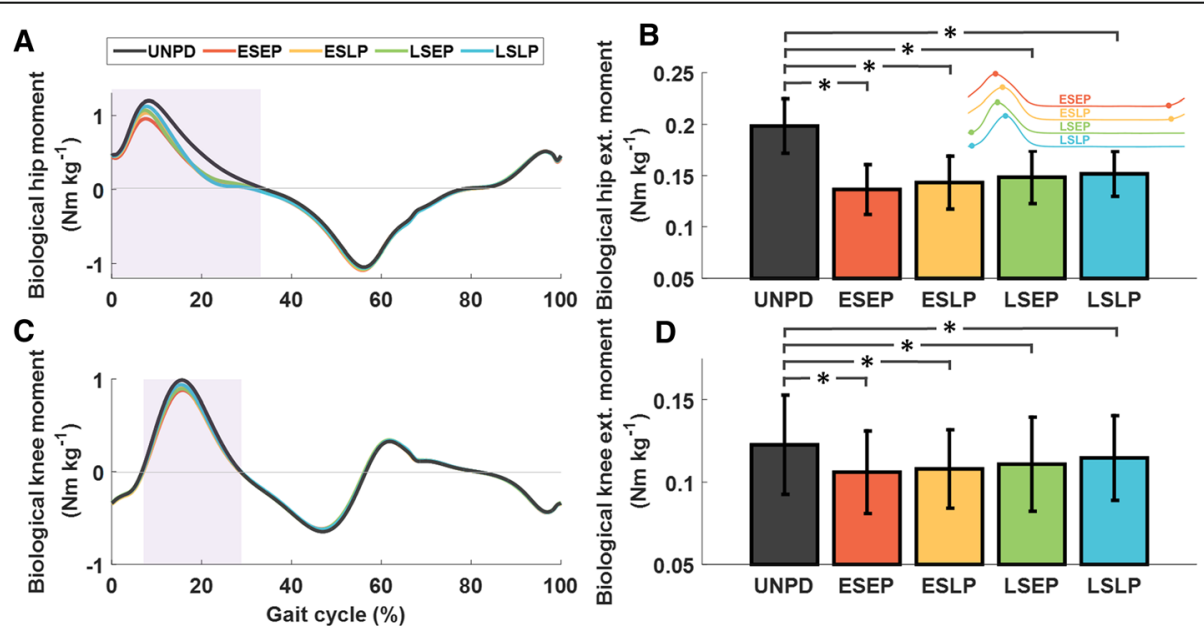

Fig. 5 a, c Average hip and knee biological joint moments of both limbs in the unpowered condition (UNPD) and in the powered conditions plotted versus gait cycle percentage. b, d Average biological knee and hip extension moment during the first half of the gait cycle (average over the shaded area in $\mathbf{a}$ and $\mathbf{c}$ ). The small panel on the right corner shows the corresponding assistive profile. Early-start-early-peak (ESEP), early-startlate-peak (ESLP), late-start-early-peak (LSEP), late-start-late-peak (LSLP) in red, yellow, green, blue respectively. The braces with * represent statistically significant differences with the unpowered condition $(p<0.050)$ 


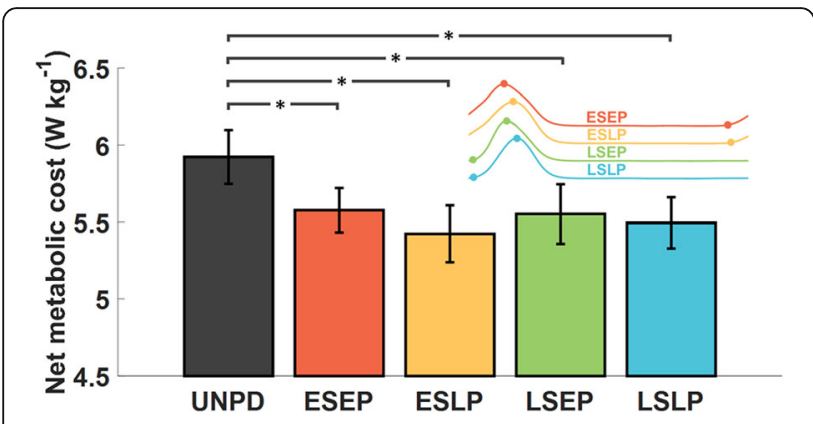

Fig. 6 Net metabolic cost reported in the unpowered condition (UNPD) and in the powered conditions. Data are means \pm SEM. The small panel on the right corner shows the corresponding assistive profile. Early-start-early-peak (ESEP), early-start-late-peak (ESLP), late-start-early-peak (LSEP), late-start-late-peak (LSLP) in red, yellow, green and blue respectively. The braces with * represent statistically significant differences with the unpowered condition $(p<0.050)$

muscle force output [52], the reduction on hip biological joint moment during the first half of the gait cycle does not only depend on muscle activation. It is likely that the lower moment may be explained by a combination of muscle length, velocity and interaction with the tendon [53]. Moreover, all the hip extensors contribute to the joint moment, while only BF was evaluated in this study. Therefore, a reduced muscle activation may not have been observed due to the small magnitude changes of each muscle. Further studies would be required to provide a more mechanistic explanation. For the subjective measurements collected in the questionnaire, we found there were no correlations between perceived assistance scores and metabolic cost. This finding suggested that it is hard to estimate metabolic cost reductions during hip assistance with a simple subjective measurement.

Last, it is worth noting a limitation in our experiment. During the testing protocol we did not include a direct comparison of an exosuit powered and a no exosuit condition, instead we compared the exosuit powered $v s$ unpowered separately from exosuit unpowered to normal walking. The reason we compared only powered $v s$ unpowered was for the practical considerations outlined in the methods section. Separate testing to evaluate the effect of wearing the suit unpowered was performed later to better understand the metabolic penalty when wearing the suit components compared to walking with a pair of pants. We found a small increase in metabolic rate $\left(0.10 \mathrm{~W} \cdot \mathrm{kg}^{-1}\right)$ but not statistically significant.

\section{Conclusions}

This study provided insight on how to manipulate the actuation timings to regulate the positive mechanical power delivered by a tethered soft exosuit assisting hip extension. Starting the assistance at terminal swing with a later peak force timing under the same magnitude of peak force allowed the soft exosuit to deliver the highest amount of positive mechanical power. This resulted in reductions in biological hip and knee power, perhaps representing a more beneficial strategy for lowering the metabolic cost. Further, reduced metabolic cost and average of the biological hip extension moment during the first half of the gait cycle were also reported for all the assistive profiles investigated in the present study, although no significant differences were reported between powered conditions. In summary, this study lays the foundation for exploration of future control strategies for autonomous hip exoskeletons designed to assist load carriers. Further, we also plan to conduct future research to explore the effect of this type of assistance on different populations.

\section{Additional file}

Additional file 1: Table S1. Questionnaire results indicated participants' perceptions on assistive conditions on a visual analogue scale from 0 to 10. Q1: "How comfortable was this active condition?" Zero indicates unbearable and 10 indicates extremely comfortable. Q2: "How did you perceive the effect of the exosuit?" Zero indicates walking is impossible and 10 indicates walking is effortless. Early-start-early-peak (ESEP), earlystart-late-peak (ESLP), late-start-early-peak (LSEP), late-start-late-peak

(LSLP). Table S2. Spatiotemporal parameters. Early-start-early-peak (ESEP), early-start-late-peak (ESLP), late-start-early-peak (LSEP), late-start-late-peak (LSLP). Data are means \pm SEM. (PDF $156 \mathrm{~kb})$

\section{Abbreviations}

ANOVA: Analysis of variance; BF: Biceps femoris; EMG: Electromyographic; ESEP: Early-start-early-peak; ESLP: Early-start-late-peak; GM: Gluteus maximum; GRFs: Ground reaction forces; IMU: Inertial measurement unit; LSEP: Latestart-early-peak; LSLP: Late-start-late-peak; MG: Medial gastrocnemius; RF: Rectus femoris; RMS: Root mean square; SEM: Standard error of the mean; SOL: Soleus; VM: Vastus medialis

\section{Acknowledgements}

The authors would like to thank Mr. Fabricio Saucedo and Mr. Stephen Allen for their help during data collection and processing.

\section{Funding}

This material is based upon the work supported by the Defense Advanced Research Projects Agency (DARPA), Warrior Web Program (Contract No. W911NF-14-C-0051). The views and conclusions contained in this document are those of the authors and should not be interpreted as representing the official policies, either expressly or implied, of DARPA or the U.S. Government. This work was also partially funded by the Wyss Institute for Biologically Inspired Engineering and the John A. Paulson School of Engineering and Applied Sciences at Harvard University.

\section{Availability of data and materials}

The dataset(s) supporting the conclusions of this article are included within the article.

\section{Authors' contributions}

YD, FAP, CS, IG and CJW designed the experiments, YD and IG designed the control and the actuation system, YD, FAP and CS performed the biomechanics experiments, YD, FAP, CS, PM, KGH and CJW analyzed and interpreted the data. All authors wrote and provided critical feedback on the manuscript. All authors read and approved the final manuscript. 


\section{Competing interests}

Patents have been filed with the U.S. Patent Office describing the soft exosuit components documented in this manuscript. YD, IG, KGH and CJW were authors of those patents. There is no other competing interest to declare.

\section{Consent for publication}

Not applicable.

\section{Ethics approval and consent to participate}

All participants provided written informed consent prior to participating in the study. Participants have provided consent for the publication of their images according to the Journal of NeuroEngineering and Rehabilitation policies. The study was approved by the Harvard Medical School Committee on Human Studies.

\section{Author details}

'John A. Paulson School of Engineering and Applied Sciences, Harvard University, 29 Oxford Street, Cambridge, MA 02138, USA. ${ }^{2}$ Wyss Institute for Biologically Inspired Engineering at Harvard, 3 Blackfan Circle, Boston, MA 02115, USA. ${ }^{3}$ Sargent College of Health and Rehabilitation Science, Boston University, 635 Commonwealth Ave, Boston, MA 02215, USA.

Received: 3 March 2016 Accepted: 27 September 2016

Published online: 03 October 2016

\section{References}

1. Walsh CJ, Endo K, Herr H. A quasi-passive leg exoskeleton for load-carrying augmentation. Int J HR. 2007:4:487-506.

2. Elliott G, Sawicki GS, Marecki A, Herr H. The biomechanics and energetics of human running using an elastic knee exoskeleton. IEEE Int Conf Rehabil Robot 2013,1-6.

3. Ferris DP, Czerniecki JM, Hannaford B. An ankle-foot orthosis powered by artificial pneumatic muscles. J Appl Biomech. 2005;21:189-97.

4. Giovacchini F, Vannetti F, Fantozzi M, Cempini M, Cortese M, Parri A, Yan T, Lefeber D, Vitiello N. A light-weight active orthosis for hip movement assistance. Rob Auton Syst. 2015;73:123-34.

5. Kitatani R, Ohata K, Takahashi H, Shibuta S, Hashiguchi Y, Yamakami N. Reduction in energy expenditure during walking using an automated stride assistance device in healthy young adults. Arch Phys Med Rehabil. 2014;95:2128-33.

6. Collins SH, Wiggin MB, Sawicki GS. Reducing the energy cost of human walking using an unpowered exoskeleton. Nature. 2015;522:212-5.

7. Malcolm P, Derave W, Galle S, De Clercq D. A simple exoskeleton that assists plantarflexion can reduce the metabolic cost of human walking. PLOS ONE. 2013;8:e56137.

8. Mooney LM, Rouse EJ, Herr HM. Autonomous exoskeleton reduces metabolic cost of human walking during load carriage. J Neuroeng Rehabil. 2014;11:80.

9. Mooney LM, Herr HM. Biomechanical walking mechanisms underlying the metabolic reduction caused by an autonomous exoskeleton. J Neuroeng Rehabil. 2016;13:4.

10. Sawicki GS, Ferris DP. A pneumatically powered knee-ankle-foot orthosis (KAFO) with myoelectric activation and inhibition. J Neuroeng Rehabil. 2009;6:23.

11. Ryder MC, Sup F. Leveraging gait dynamics to improve efficiency and performance of powered hip exoskeletons. IEEE Int Conf Rehabil Robot 2013,1-6.

12. Shamaei K, Cenciarini M, Adams AA, Gregorczyk KN, Schiffman JM, Dollar AM. Design and evaluation of a quasi-passive knee exoskeleton for investigation of motor adaptation in lower extremity joints. IEEE Trans Biomed Eng. 2014;61:1809-21.

13. van Dijk W, van der Kooij H, Hekman E. A passive exoskeleton with artificial tendons: Design and experimental evaluation. IEEE Int Conf Rehabil Robot 2011, 1-6.

14. Berkeley Bionics introduces eLEGS robotic exoskeleton, http://spectrum.ieee. org/automaton/robotics/medical-robots/berkeley-bionics-introduces-elegsrobotic-exoskeleton. 1 Oct 2016.

15. Blaya JA, Herr H. Adaptive control of a variable-impedance ankle-foot orthosis to assist drop-foot gait. IEEE Trans Neural Syst Rehabil Eng. 2004;12:24-31.

16. Esquenazi A, Talaty M, Packel A, Saulino M. The ReWalk powered exoskeleton to restore ambulatory function to individuals with thoracic-level motor-complete spinal cord injury. Am J Phys Med Rehabil. 2012;91:911-21.
17. Hollander KW, llg R, Sugar TG, Herring D. An efficient robotic tendon for gait assistance. J Biomech Eng. 2006;128:788-91.

18. Hu J, Lim YJ, Ding Y, Paluska D, Solochek A, Laffery D, Bonato P, Marchessault R. An advanced rehabilitation robotic system for augmenting healthcare. Int Conf IEEE Eng Med Biol Soc. 2011;2011:2073-6.

19. Jezernik S, Colombo G, Keller T, Frueh H, Morari M. Robotic orthosis Lokomat: a rehabilitation and research tool. Neuromodulation. 2003;6:108-15.

20. Kawamoto H, Hayashi T, Sakurai T, Eguchi K, Sankai Y. Development of single leg version of HAL for hemiplegia. Conf IEEE Eng Med Biol Soc. 2009;2009:5038-43.

21. Lenzi T, Carrozza MC, Agrawal SK. Powered hip exoskeletons can reduce the user's hip and ankle muscle activations during walking. IEEE Trans Neural Syst Rehabil Eng. 2013;21:938-48.

22. Olivier J, Bouri M, Ortlieb A, Bleuler H, Clavel R. Development of an assistive motorized hip orthosis: Kinematics analysis and mechanical design. IEEE Int Conf Rehabil Robot. 2013;2013:1-5.

23. Neuhaus PD, Noorden JH, Craig TJ, Torres T, Kirschbaum J, Pratt JE. Design and evaluation of Mina: a robotic orthosis for paraplegics. IEEE Int Conf Rehabil Robot 2011,1-8.

24. Ronsse R, Lenzi T, Vitiello N, Koopman B, Van Asseldonk E, De Rossi SMM, Van Den Kieboom J, Van Der Kooij H, Carrozza MC, ljspeert AJ. Oscillatorbased assistance of cyclical movements: model-based and model-free approaches. Med Biol Eng Comput. 2011;49:1173-85.

25. Veneman JF, Kruidhof R, Hekman EEG, Ekkelenkamp R, Van Asseldonk EHF, Van Der Kooij H. Design and evaluation of the LOPES exoskeleton robot for interactive gait rehabilitation. IEEE Trans Neural Syst Rehabil Eng. 2007;15:379-86.

26. Asbeck AT, De Rossi SMM, Holt KG, Walsh CJ. A biologically inspired soft exosuit for walking assistance. Int J Rob Res. 2015;34:744-62.

27. Asbeck AT, De Rossi SMM, Galiana I, Ding Y, Walsh CJ. Stronger, smarter, softer: next generation wearable robots. IEEE Robot Autom. 2014;21:22-33.

28. Ding Y, Galiana I, Asbeck AT, De Rossi SMM, Bae J, Teles Dos Santos TR, Lara De Araujo V, Lee S, Holt K, Walsh CJ. Biomechanical and physiological evaluation of multi-joint assistance with soft exosuits. IEEE Trans Neural Syst Rehabil Eng. 2016;9:1-11.

29. Ding Y, Galiana I, Siviy C, Panizzolo FA, Walsh CJ. IMU-based iterative control for hip extension assistance with a soft exosuit. IEEE Int Conf Robot Autom 2016,3501-8.

30. Panizzolo FA, Galiana I, Asbeck AT, Siviy C, Schmidt K, Holt KG, Walsh CJ. A biologically-inspired multi-joint soft exosuit that can reduce the energy cost of loaded walking. J Neuroeng Rehabil. 2016;13:43.

31. Farris DJ, Sawicki GS. The mechanics and energetics of human walking and running: a joint level perspective. J R Soc Interface. 2011;9:110-8.

32. Sawicki GS, Lewis $\mathrm{CL}$, Ferris DP. It pays to have a spring in your step. Exerc Sport Sci Rev. 2009;37:130-8.

33. Winter DA. Energy generation and absorption at the ankle and knee during fast, natural, and slow cadences. Clin Orthop Relat Res. 1983;175:147-54.

34. Dean JC, Kuo AD. Elastic coupling of limb joints enables faster bipedal walking. J R Soc Interface. 2009;6:561-73.

35. Kuo AD. Energetics of actively powered locomotion using the simplest walking model. J Biomech Eng. 2001;124:113-20.

36. Wang S, van Dijk W, van der Kooij H. Spring uses in exoskeleton actuation design. IEEE Int Conf Rehabil Robot. 2011;2011:1-6.

37. Lewis $\mathrm{CL}$, Ferris $\mathrm{DP}$. Invariant hip moment pattern while walking with a robotic hip exoskeleton. J Biomech. 2011;44:789-93.

38. Jackson RW, Collins SH. An experimental comparison of the relative benefits of work and torque assistance in ankle exoskeletons. J Appl Physiol. 2015;119:541-57.

39. Wehner M, Quinlivan B, Aubin PM, Martinez-Villalpando E, Baumann M, Stirling L, Holt K, Wood R, Walsh C. A lightweight soft exosuit for gait assistance. IEEE Int Conf Robot Autom. 2013,3362-9.

40. Hasaneini SJ, Macnab CJB, Bertram JEA, Leung H. Optimal relative timing of stance push-off and swing leg retraction. IEEE Int Conf Intell Robot Syst. 2013,3616-23.

41. Umberger BR, Rubenson J. Understanding muscle energetics in locomotion. Exerc Sport Sci Rev. 2011;39:59-67.

42. Sawicki GS, Ferris DP. Powered ankle exoskeletons reveal the metabolic cost of plantar flexor mechanical work during walking with longer steps at constant step frequency. J Exp Biol. 2009;212:21-31. 
43. Knapik J, Harman E, Reynolds K. Load carriage using packs: a review of physiological, biomechanical and medical aspects. Appl Ergon. 1996;27:207-16

44. Ding Y, Galiana I, Asbeck A, Quinlivan B, De Rossi SMM, Walsh C. Multi-joint actuation platform for lower extremity soft exosuits. IEEE Int Conf Robot Autom. 2014,1327-34.

45. Winter DA. Biomechanics and motor control of human movement. Waterloo: University of Waterloo Press; 1991

46. Perry J, Burnfield JM. Gait analysis: normal and pathological function Thorofare: SLACK Incorporated; 2010.

47. Brockway JM. Derivation of formulae used to calculate energy expenditure in man. Hum Nutr Clin Nutr. 1987;41:463-71.

48. Hermens HJ, Freriks B, Disselhorst-Klug C, Rau G. Development of recommendations for SEMG sensors and sensor placement procedures. J Electromyogr Kinesiol. 2000:10:361-74.

49. Sawicki GS, Ferris DP. Mechanics and energetics of level walking with powered ankle exoskeletons. J Exp Biol. 2008:211:1402-13.

50. Grabowski AM. Metabolic and biomechanical effects of velocity and weight support using a lower-body positive pressure device during walking. Arch Phys Med Rehabil. 2010;91:951-7.

51. Farris DJ, Robertson BD, Sawicki GS. Elastic ankle exoskeletons reduce soleus muscle force but not work in human hopping. J Appl Physiol. 2013;115:579-85.

52. Roberts TJ, Gabaldón AM. Interpreting muscle function from EMG: lessons learned from direct measurements of muscle force. Integr Comp Biol. 2008:48:312-20.

53. Zajac FE. Muscle and tendon: properties, models, scaling, and application to biomechanics and motor control. Crit Rev Biomed Eng. 1989;17:359-411.

\section{Submit your next manuscript to BioMed Central and we will help you at every step:}

- We accept pre-submission inquiries

- Our selector tool helps you to find the most relevant journal

- We provide round the clock customer support

- Convenient online submission

- Thorough peer review

- Inclusion in PubMed and all major indexing services

- Maximum visibility for your research

Submit your manuscript at www.biomedcentral.com/submit

C) Biomed Central 